\title{
SUPERCONDUCTORS
}

\section{Unusual oxygen isotope effects in cuprates?}

\section{Arising from: G.-H. Gweon et al. Nature 430, 187-190 (2004)}

The possibility that a pairing boson might act as the 'glue' to bind electrons into a Cooper pair in superconductors with a high critical temperature $\left(T_{c}\right)$ is being actively pursued in condensed-matter physics. Gweon et al. ${ }^{1}$ claim that there is a large and unusual oxygen-isotope effect on the electronic structure, indicating that phonons have a special importance in high-temperature superconductors. However, we are unable to detect this unusual oxygenisotope effect in new data collected under almost identical material and experimental conditions. Our findings point towards a more conventional influence of phonons in these materials.

In the high-temperature superconductors, oxygen-isotope exchange has been found to have a very small effect on the $T_{c}$, particularly for optimally doped (highest- $T_{\mathrm{c}}$ ) samples ${ }^{2}$. It was therefore unexpected when Gweon et al. reported a large isotope effect on the electronic structure in optimally doped $\mathrm{Bi}_{2} \mathrm{Sr}_{2} \mathrm{CaCu}_{2} \mathrm{O}_{8+\delta}$ using angle-resolved photoemission spectroscopy (ARPES) ${ }^{1}$. This isotope effect was seen mainly at a broad, high-energy hump feature and was unusually large (10-40 meV shift), by contrast with the expected isotopic phonon shifts of the order of $3 \mathrm{meV}$. It was argued by many that this is experimental evidence that the coupling between the lattice and electrons plays a particularly significant role.

Figure 1a shows our energy-momentum $(E-k)$ dispersion relations for both ${ }^{16} \mathrm{O}$ and ${ }^{18} \mathrm{O}$ taken along the 'nodal' direction of the Brillouin zone. The results show an insignificant shift for the deeper states of $-0.9 \pm 0.4 \mathrm{meV}$, by contrast with Fig. 1b of Gweon et al. ${ }^{1}$, which shows a $15-\mathrm{meV}$ shift of more deeply lying states. We found that precise rotational alignment of the samples was crucial to obtain repeatable energy positions, especially in the high-energy regime discussed here. The negligible energy shift was confirmed by using three different facilities, samples with multiple doping levels, including the optimal doping described in ref. 1 , and with various photon energies, including the same energy used by Gweon et al. ${ }^{1}(33 \mathrm{eV})$, and a low photon energy $(7.0 \mathrm{eV})$ that gives extreme resolution, as well as increased bulk sensitivity.

Gweon et al. ${ }^{1}$ show an even larger effect (up to $40 \mathrm{meV}$ ) at the 'antinodal' portions of the Brillouin zone (towards the $(0, \pi)$ and $(0,-\pi)$ points) - comparable data are shown here in Fig. 1b. We studied ten cuts that had a similar geometry to the key cut 7 of Gweon et al. ${ }^{1}$, with half of the cuts at positive $k_{y}$ (momentum along the $y$ direction) values and the others at negative $k_{y}$. Having data on both sides of the $(0,0)$ point allowed the correction of any very slight ( 0.1 degrees) sample misalignments, which is crucial for removing systematic alignment errors that could cause apparent energy shifts. Figure $1 \mathrm{~b}$ (right) shows the dispersion of the bottom of the band (determined in each cut as in Fig. $2 \mathrm{~d}$ of Gweon et al. $\left.{ }^{1}\right)$ along the $(0,-\pi) \rightarrow$ $(0, \pi)$ symmetry line. The ten sets in Fig. $1 \mathrm{~b}$ have an average isotope shift of the band bottom of $2 \pm 3 \mathrm{meV}$, which is inconsistent with the 10-40 meV level found by Gweon et al. ${ }^{1}$.

Our result is not inconsistent with the tunnelling result of a conventional phononic isotope shift on the scale of about $3 \mathrm{meV}$ (refs 3,4) that was performed on the same samples and which conceivably could be seen near the kink energy in future ultra-resolution ARPES.
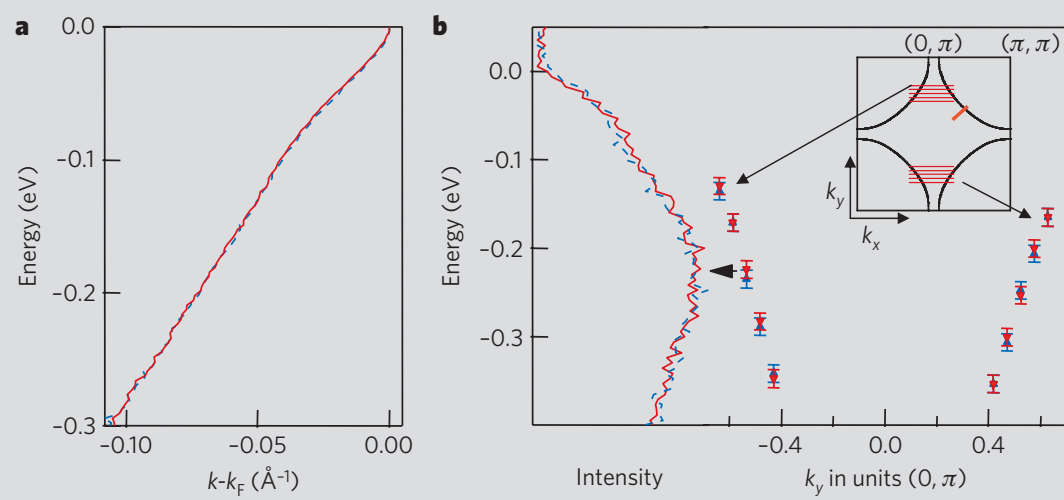

Figure 1 Comparison of nodal and antinodal energy $(E)$ and momentum $(k)$ dispersions as a function of oxygen-isotope exchange. All data are from optimally doped $\left(T_{\mathrm{c}}=92 \mathrm{~K}\right.$, unexchanged $)$ $\mathrm{Bi}_{2} \mathrm{Sr}_{2} \mathrm{CaCu}_{2} \mathrm{O}_{8+\delta}$ crystals taken with $33 \mathrm{eV}$ photons at $15 \mathrm{~K}$. Blue line and symbols, ${ }^{16} \mathrm{O}$ data; red line and symbols, ${ }^{18} \mathrm{O}$ data. No large-scale shift of the spectra with isotope exchange is evident. a, Nodal dispersion (red diagonal line in $\mathbf{b}$, inset) obtained by fitting momentum-distribution curves, in which $k_{\mathrm{F}}$ is the Fermi momentum. $\mathrm{b}$ (left), Energy-distribution curve from each isotope at the $k$-point indicated (arrowhead); right, dispersion of the band bottom from energy-distribution peaks along the $(0,-\pi)-(0, \pi)$ symmetry line (horizontal lines in the Fermi surface inset).

Although we do not observe the unusual largescale isotope effect reported by Gweon et al. ${ }^{1}$, our result does not invalidate electron-phonon coupling as a potential pairing mechanism for high-temperature superconductivity. Rather, it implies that if electron-phonon coupling is responsible, it must do it in a more subtle, roundabout way.

Methods. Our data were measured with 33-eV photons at BL12 of the Advanced Light Source, Berkeley, USA, by use of a high-precision 6axis sample manipulator. Single crystals of $\mathrm{Bi}_{1.9} \mathrm{Sr}_{2.1} \mathrm{CaCu}_{2} \mathrm{O}_{8+\delta}$ were annealed in either ${ }^{18} \mathrm{O}_{2}$ or ${ }^{16} \mathrm{O}_{2}$ gas under the same thermal conditions: $800{ }^{\circ} \mathrm{C}$ at 1 atmosphere for $168 \mathrm{~h}$, followed by $700{ }^{\circ} \mathrm{C}$ at 0.2 bar for $24 \mathrm{~h}$. The $T_{\mathrm{c}}$ values of the samples decreased from $92 \mathrm{~K}$ to $91 \mathrm{~K}$ on isotope substitution. Raman spectra showed a clear shift of the $B_{1 \mathrm{~g}}$ vibration modes, confirming that isotope substitution was nearly complete at $80 \%$ or more.

John F. Douglas ${ }^{\star}$, Hideaki Iwasawa $†$, Zhe Sun*, Alexei V. Fedorov\$, Motoyuki Ishikadoๆ, Tomohiko Saitoh $\uparrow$, Hiroshi Eisaki Hiroshi Bandoł, Takeshi Iwase $\%$, Akihiro Ino||, Masashi Arita\#, Kenya Shimada\#, Hirofumi Namatame\#, Masaki Taniguchi||\#, Takahiko Masui ${ }^{\star \star}$, Setsuko Tajima ${ }^{\star \star}$, Kazuhiro Fujitaף, Shin-ichi Uchidaๆ, Yoshihiro Aiurał, Daniel S. Dessau*

*Department of Physics, University of Colorado, Boulder, Colorado 80309-0390, USA

†Department of Applied Physics, Tokyo University of Science, Tokyo 162-8601, Japan

National Institute of Advanced Industrial Science and Technology, Tsukuba, Ibaraki 305-8568, Japan §Advanced Light Source, Lawrence Berkeley Lab, Berkeley, California 94720, USA.

ๆDepartment of Physics, University of Tokyo, Tokyo 113-8656, Japan

||Graduate School of Science, Hiroshima

University, Higashi-Hiroshima 739-8526, Japan \#Hiroshima Synchrotron Radiation Center, Hiroshima University, Higashi-Hiroshima 7390046, Japan

**Department of Physics, Osaka University, 1-1 Machikaneyama, Toyonaka, Osaka 560-0043, Japan

e-mail:y.aiura@aist.go.jp

Received 29 September 2006; accepted 15 February 2007.

1. Gweon, G.-H. et al. Nature 430, 187-190 (2004).

2. Franck, J. P. in Physical Properties of High Temperature Superconductors IV (ed. Ginsberg, D. M.) 189-293 (World Scientific, Singapore, 1994).

3. Lee, J. et al. Nature 442, 546-550 (2006).

4. de Lozanne, A. Nature 442, 522-523 (2006).

Competing financial interests: declared none. doi: $10.1038 /$ nature 05738 